\title{
Comparison of Linear and Non-Linear Equalizer using the Maltlab
}

\author{
Seema Paliwal \\ Assitant professor \\ SDITS \\ Khandwa
}

\author{
Dilpreet Kaur Grover \\ Under graduate student of \\ engg. \\ SDITS Khandwa
}

\author{
Jyoti Krayla \\ Under graduate student of \\ engg. \\ SDITS Khandwa
}

\begin{abstract}
In this paper, we compare the BER performance, burst error performance and the signal power spectrum of different types of equalizer using the MATLAB simulation. The simulation and results shows the superiority of equalizers on one another.
\end{abstract}

\section{General Terms}

Inter symbol interference, signal to noise ratio.

\section{Keywords}

Bit error rate, equalizer, burst error.

\section{INTRODUCTION}

In digital communication, the ultimate goal is the reliable transmission of information at the highest possible data rates. But inter symbol interference (ISI) occur when the high speed data is transmitted over the communication channel.

When the signal is transmitted from source, while reaching to destination, a delay spread is generated in all the multipath objects (IOs) which results in ISI and we get distorted signal at the receiver end. So, for the reliable communication we have to do some advancement at the receiver side which mitigates the effect of ISI.

For credible communication, we have to use equalization techniques at the receiver side to combat ISI. An equalization technique is that which compensate ISI created by multipath tine dispersive or time varying channels. So, the main purpose of equalizer is to reverse the effect that channel has on the transmitted signal, with the main aim of reproducing the original signal at the receiver end.

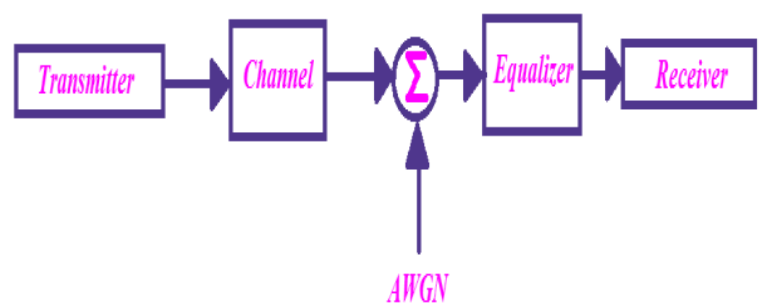

Fig.1 System Model

Fig.1 shows the system model where a transmitter sends information through a dispersive channel due to which the channel output is corrupted by the additive white Gaussian noise. The task of the equalizer is to eliminate the effect of channel from the transmitted information and also to recover it and hence we get the original signal at the output of the receiver.

\section{EQUALIZATION TECHNIQUES}

There are two types of equalization techniques:

- Linear Equalization- Suboptimal, but simple.

- Non-linear Equalization- for severe and noisy channels.

\subsection{Linear Equalizer}

The most simple and common type of channel equalizer used in practice to reduce the ISI is a linear equalizer. The linear equalizer can be implemented as an FIR filter also known as transversal filter. In this equalizer, the current and the past values of the received signal are linearly weighted by the adjustable filter coefficient and summed to produce the output as shown in Fig2.

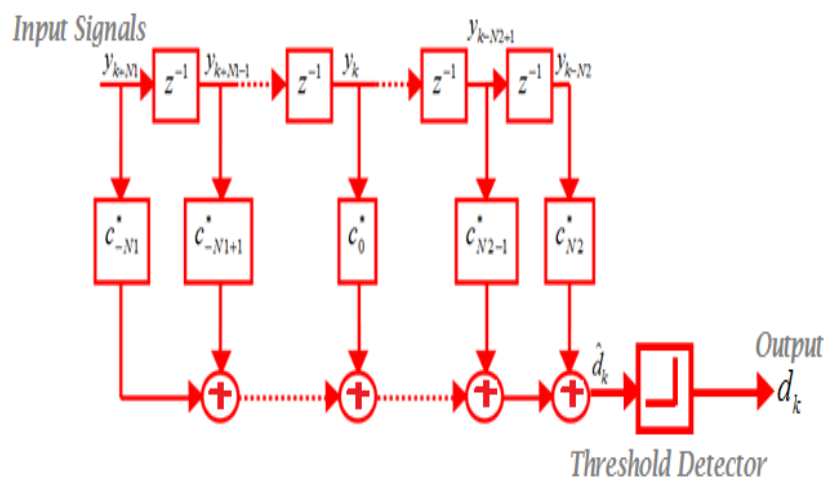

Fig.2 Structure of a linear transversal equalizer.

The minimum mean squared error that a linear equalizer can achieve is:

$$
E\left[|e(n)|^{2}\right]=\frac{T}{2 \pi} \int_{-\pi / T}^{\pi / T} \frac{N_{0}}{\mid F\left(\left.e^{j \omega T}\right|^{2}+N_{0}\right.} d \omega
$$

\subsection{Non-linear Equalizer}

Non-linear equalizers are used in applications where the channel distortion is to severe for a linear equalizer to mitigate the effect of channel impairments. The reason for choosing non-linear equalizers over linear equalizer is that the latter's performance in channel that exhibit nulls is not effective. Noise enhancement in these regions and long impulse response are a problem. The basic reason for this problem is that in linear filtering and noise are processed together, causing noise enhancement problem.

Based upon the importance, the noise-linear are classified as: 
- Decision Feedback Equalizer (DFE).

- Maximum Likelihood Sequence Estimation (MLSE).

\subsubsection{Decision feedback equalizer}

A decision feedback equalizer is a simple non-linear equalizer, particularly useful for channel with severe amplitude distortion. DFE consist of a feed forward filter (FFF) and feedback filter (FBF). The Fig3 shows the block diagram of decision feedback equalizer. The feed forward section is nothing but a linear equalizer whose output is given to the decision device. The feedback section is driven by the output of the decision device.

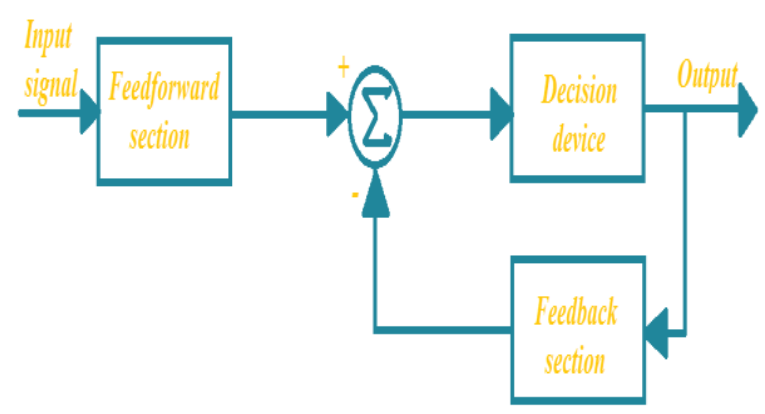

Fig.3 Block diagram of decision feedback equalizer.

The basic idea behind DFE is that once we have detected information symbol a decided upon, the ISI that induces on the future symbols can be estimated an subtracted out before detection of subsequent symbols.

The minimum mean squared error that a decision feedback equalizer can achieve is:

$$
E\left[|e(n)|^{2}\right]=\exp \left\{\frac{T}{2 \pi} \int_{-\pi / T}^{\pi / T} \ln \left[\frac{N_{0}}{\mid F\left(\left.e^{j \omega T}\right|^{2}+N_{0}\right.}\right] d \omega\right\}
$$

The minimum mean squared error of decision feedback equalizer is smaller than that of a linear equalizer.

\subsubsection{Maximum Likehood Sequence Estimation}

A DFE is not an optimum equalizer because it just outmatches the linear equalizer. MLSE gives optimum performance as it tests all the possible data sequences and choose that data as output which has the maximum probability. MLSE as an equalizer was first proposed by Forney [For78] in which he setup a basic estimator structure and implement it with Viterbi algorithm. However, the computational complexity of an MLSE increases with large delay spread and signal constellation size. The number of states of the Viterbi decoder is expressed as $\mathrm{M}^{\mathrm{L}}$, where $\mathrm{M}$ is the number of symbols in constellation, and $\mathrm{L}$ is the channel-speed length.

The block diagram of MLSE receiver based on DFE is shown in Fig4. The MLSE is optimal in the sense that it minimized the probability of a sequence error.

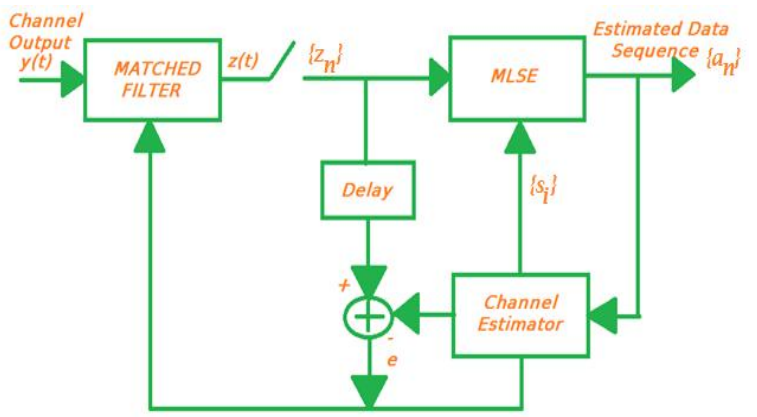

Fig.4 Block diagram of MLSE.

The MLSE requires the knowledge of-

- The channel characteristics in order to compute the metrics for making decisions.

- The statistical distribution of the noise corrupting the signal.

\section{BIT ERROR RATE}

When the bits stream transmitted from the source over the communication channel, then the number of bits received that altered due to noise, interference, distortion or bit synchronization errors is the number of bit errors.

Hence, BER is a performance measurement that specifies the number of bit corrupted or destroyed as they are transmitted from its source to its destination or BER is the number of bit error per unit time. BER can also be defined in terms of the probability of error (POE).

By the definition of bit error rate we can define its simple formula:

$$
\text { BER }=\frac{\text { No. of Errors }}{\text { Total no. of bits sent }}
$$

There are several factors that affect BER include bandwidth, signal to noise ratio (SNR), transmission speed and transmission medium.

\section{SIGNAL TO NOISE RATIO}

Signal to noise ratio (SNR) is a measure of the amount of signal divided by the amount of noise being received. SNR is mathematically expressed as:

$$
\mathrm{SNR}=10 \log _{10}\left(\mathrm{E}_{\mathrm{b}} / \mathrm{N}_{0}\right) \mathrm{dB}
$$

where, $\left(\mathrm{E}_{\mathrm{b}} / \mathrm{N}_{0}\right)$ is the normalized SNR. Normalized SNR is the ratio of energy per bit to noise power spectral density or it is also called SNR per bit.

A high Signal to Noise ratio is good because it means that we are getting more signal and less noise. If SNR is high then the strength of the signal is also high but if it is low or very poor then the signal is totally distorted and we can't recover the original signal from it.

\section{BURST ERROR}

In telecommunication, a contiguous sequence of symbol is transmitted through the data transmission channel, and when they are opened at the receiver end the first and the last symbol are in error and there exist no contiguous sequence of 
"m" symbols between them, this is the burst error. The integer parameter " $\mathrm{m}$ " is referred to as guard band of the error burst.

\section{SIMULATION AND RESULT}

Using MATLAB simulation we get following results for different equalizer such as linear, decision feedback and MLSE in the terms of BER and Signal to Noise ratio and Burst error occurrence.

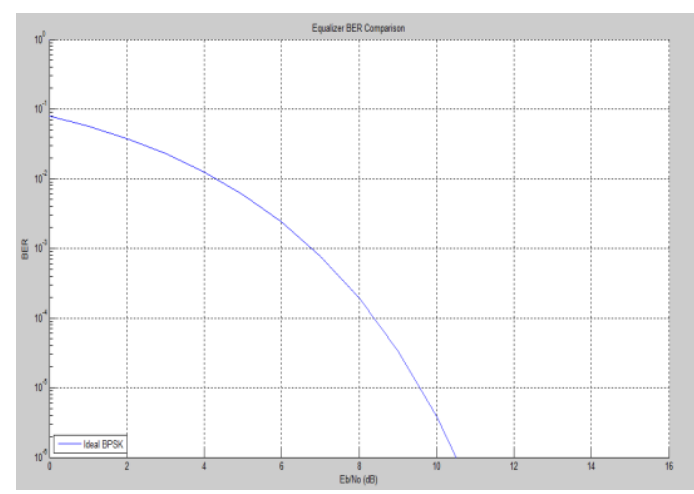

Fig.5: Unequalized BER performance

Fig. 5 shows Unequalized BER performance. When the signal is transmitted from source, then at the receiver side we haven't used the equalizer, i.e. the output which we get at the receiver end is unequalized output. In this figure as the SNR increases the BER decreases.

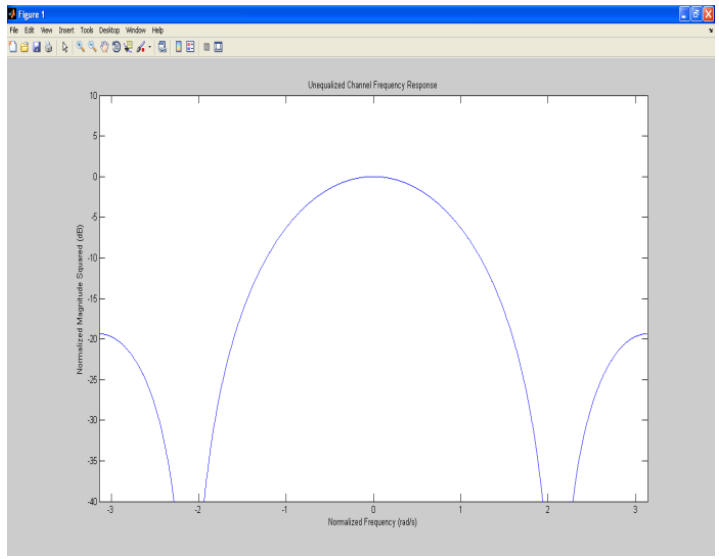

Fig.6: Unequalized Channel Frequency Response

Fig.6 shows the unequalized signal power spectrum. In this figure there are very deep nulls which means that the channel is to severe and the signal can be strongly distorted by ISI. But in this the main lobe has very high amplitude and side lobes have very small amplitudes which means that $99.9 \%$ information is contained by main lobe.

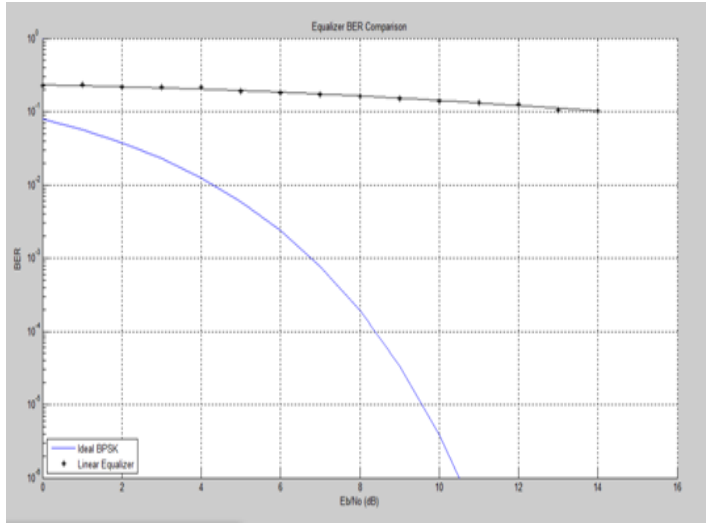

Fig.7: Comparison between Ideal BPSK and linear equalizer

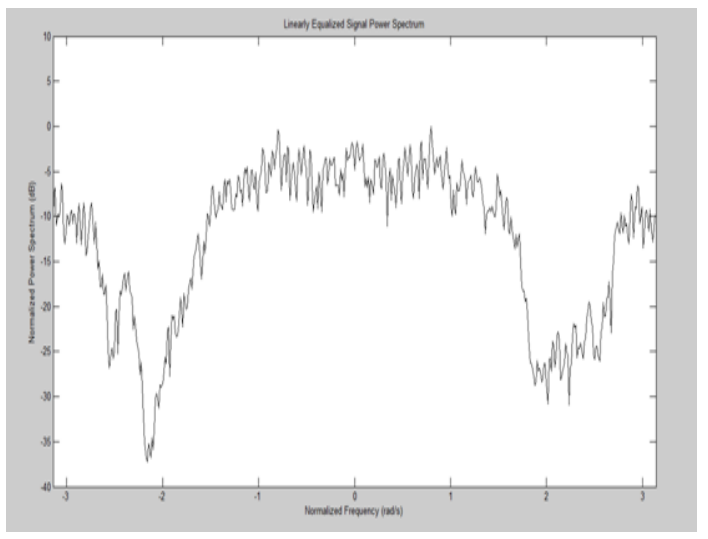

Fig.8: Linear Equalizer Signal Power Spectrum

Fig.8: Linear Equalizer Signal Power Spectrum. Here we find that the amplitude of side lobes are increases but the deepness of nulls decreases.

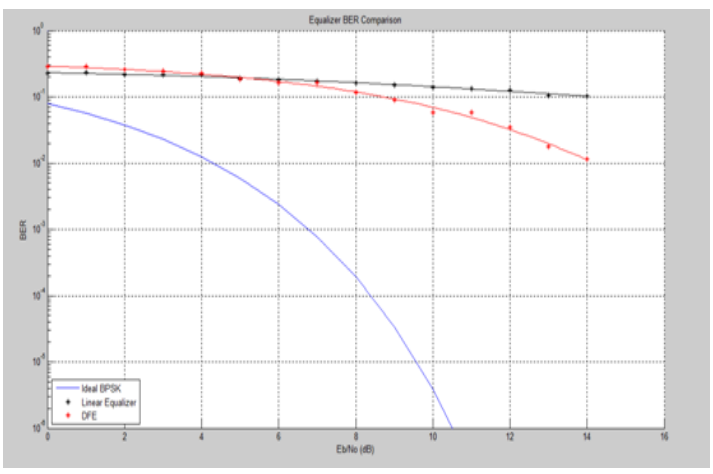

Fig.9: Comparison among BPSK, Linear and DFE equalizer

Fig.9 shows Comparison among BPSK, Linear and DFE equalizer. On comparing the DFE output with the linear equalizer output we get improved BER performance, i.e. as the SNR increases the BER of the DFE decreases more rapidly as compared to the linear equalizer. 


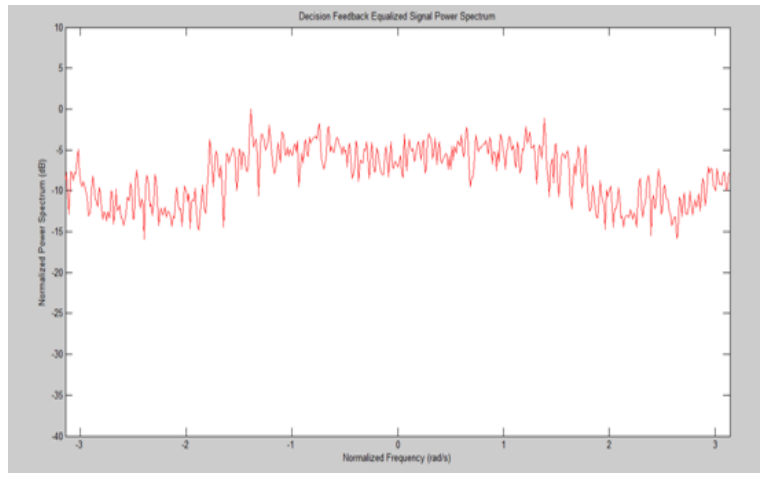

Fig.10: DFE Signal Power Spectrum

Fig.10 shows DFE Signal Power Spectrum. Here the nulls are less deep and the magnitude of side lobes is also small

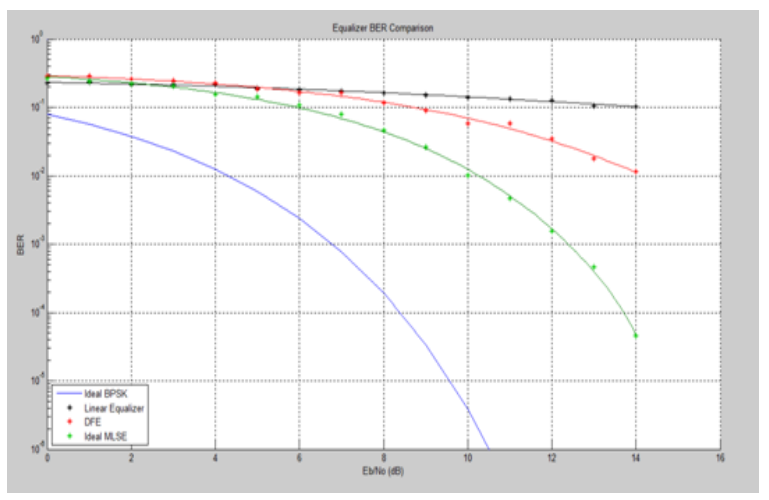

Fig.11: Comparison among BPSK, Linear, DFE and Ideal MLSE equalizer.

Fig.11 shows Comparison among BPSK, Linear, DFE and Ideal MLSE equalizer. On the analysis basis of BER vs SNR performance we found that the BER performance of ideal MLSE is better than that of others.

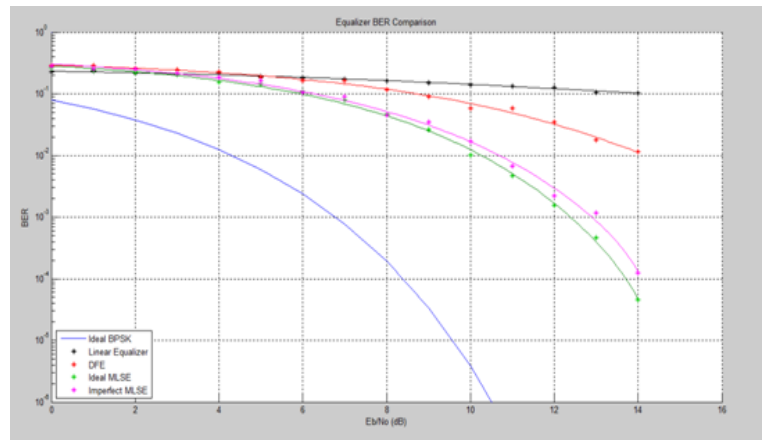

Fig.12: Comparison among BPSK, Linear, DFE,Ideal MLSE and Imperfect MLSE equalizer.

Fig.12 shows Comparison among BPSK, Linear, DFE,Ideal MLSE and Imperfect MLSE equalizer. Here the BER performance of the imperfect MLSE is fairly closely to that of ideal MLSE. For ideal MLSE we assume that the channel is known and for imperfect MLSE the channel is time varying.

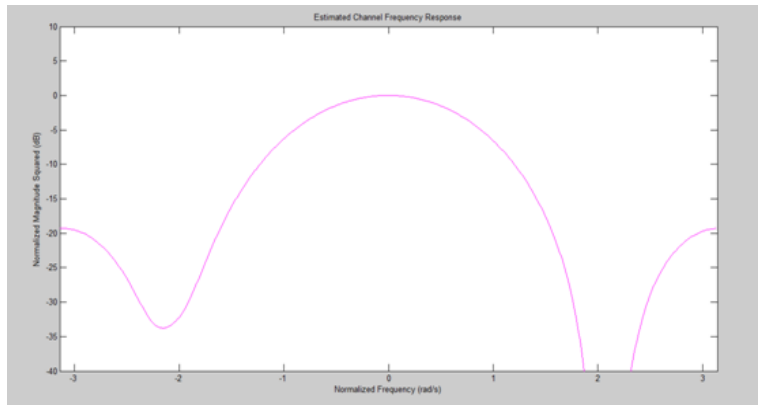

Fig.13: Imperfect MLSE Channel Frequency Response

Fig.13 shows Imperfect MLSE Channel Frequency Response. Here, the nulls are deep but the magnitude of the main lobe is very high. So it

\section{CONCLUSION}

This paper deals with the detailed performance analysis of linear equalizer and non-linear equalizer.

On the basis of theory we conclude the following -

- Linear equalizer is best suited for comparatively flat channel spectrum.

- When the channel distortion is too severe for the linear equalizer to mitigate the effects of channel impairments then we use decision feedback equalizer.

DFE reach to pretty good steady-state performance even with severe and noisy channels. But as DFE use decisions on data for removing the part of ISI; incorrect decisions can cause propagation error in DFEs, since an incorrect decision may add ISI instead of removing it.

The minimum mean square error of DFE is always less than that of linear equalizer.

- Maximum likelihood sequence estimation (MLSE) is optimal in the sense of having the lowest probability of detecting the wrong sequence.

After emerging the above simulation and result we conclude the following:

- As the simulation progresses, the BER performance updates for comparative analysis between the equalization techniques, i.e. the BER performance of MLSE is better than others equalizers.

- The signal power spectrum of DFE is much better than that of linear equalizer.

- At the low BERs, both the MLSE algorithm and the DFE algorithm suffer from error bursts.

Table 1. BER performance of different Equalizer

\begin{tabular}{|c|c|c|c|c|c|}
\hline $\mathrm{E}_{\mathrm{b}} / \mathrm{N}_{0}$ & $\begin{array}{c}\text { Unequali } \\
\text { zed } \\
\text { Output }\end{array}$ & $\begin{array}{c}\text { Linear } \\
\text { Equaliz } \\
\text { er } \\
\text { Output }\end{array}$ & $\begin{array}{c}\text { DFE } \\
\text { Outp } \\
\text { ut }\end{array}$ & $\begin{array}{c}\text { Ideal } \\
\text { MLSE } \\
\text { Equaliz } \\
\text { er } \\
\text { Output }\end{array}$ & $\begin{array}{c}\text { Imperf } \\
\text { ect } \\
\text { MLSE } \\
\text { Equaliz } \\
\text { er } \\
\text { Output }\end{array}$ \\
\hline 0 & 0.0786 & 0.2328 & 0.29 & 0.2522 & 0.3057 \\
\hline 1 & 0.0562 & 0.2265 & 0.27 & 0.2462 & 0.2774 \\
\hline
\end{tabular}




\begin{tabular}{|c|c|c|c|c|c|}
\hline & & & 73 & & \\
\hline 2 & 0.0375 & 0.2196 & $\begin{array}{c}0.26 \\
03\end{array}$ & 0.2253 & 0.2462 \\
\hline 3 & 0.0228 & 0.2195 & $\begin{array}{c}0.24 \\
11\end{array}$ & 0.2126 & 0.2156 \\
\hline 4 & 0.0125 & 0.2118 & $\begin{array}{c}0.20 \\
35\end{array}$ & 0.1775 & 0.1779 \\
\hline 5 & 0.0059 & 0.1963 & $\begin{array}{c}0.19 \\
45\end{array}$ & 0.1311 & 0.1630 \\
\hline 6 & 0.0023 & 0.1849 & $\begin{array}{c}0.17 \\
13\end{array}$ & 0.1084 & 0.1084 \\
\hline 7 & 0.0007 & 0.1748 & $\begin{array}{c}0.14 \\
51\end{array}$ & 0.0683 & 0.0775 \\
\hline 8 & 0.0001 & 0.1643 & $\begin{array}{c}0.11 \\
87\end{array}$ & 0.0453 & 0.0514 \\
\hline 9 & $3.3 \mathrm{e}^{-005}$ & 0.1539 & 0.09 & 0.0310 & 0.0348 \\
& & & 31 & & \\
\hline 10 & $3.8 \mathrm{e}^{-006}$ & 0.1426 & $\begin{array}{c}0.06 \\
95\end{array}$ & 0.0114 & 0.0166 \\
\hline
\end{tabular}

[2] T. S. Rappaport, Wireless Communication, Chapter 5, second edition Upper Saddle River, NJ: Prentice Hall, 1996.

[3] T. S. Rappaport, Wireless Communication, Chapters. 3 and 4, UpperSaddle River, NJ: Prentice Hall, 1996.

[4] BER performance analysis of MIMO system using Equalization technique by Kirthi Aparna Tella UFID 23611267

[5] A. T. Erdogan, B. Hassibi, and T. Kailath, - H1 Equalization of Communication Channels, $\|$ submitted to IEEE Tr. Signal Pr., 1997. [6] E. A. Lee, D. G. Messerschmitt, \|Digital communications\|, 2nd Edn., Kluwer Academic Publishers, 1994.

[6] A. T. Erdogan, B. Hassibi, and T. Kailath, - H1 Equalization of Communication Channels, $\|$ submitted to IEEE Tr. Signal Pr., 1997.

[7] Adaptive Bayesian Decision Feedback Equalizer for Alpha-Stable Noise Environment, Apostolos T. Georgiadis and Bernard Mulgrew, University of Einburgh, EH9 3JL Edinburgh, UK.

[8] https://en.wikipedia.org/wiki/Equalizer

\section{REFERENCES}

[1] J. G. Proakis, \|Digital Communications", New York: McGraw-Hill, 2000 